Boletín de la Sociedad Botánica de México 48: 135-138, 1988

DOI: $10.17129 /$ botsci.1351

Bol. Soc. Bot. México 48: 135-138 (1988)

\title{
Spigelia longiflora (Mart. et. Gal.): actividad antialimentaria en tres especies de insectos fitófagos
}

\author{
Miguel Ángel Villavicencio y Blanca E. Pérez Escandón ${ }^{1}$
}

RESUMEN. Se probó la actividad antialimentaria en insectos de una fracción obtenida de hojas de Spigelia longiflora (Loganiaceae) utilizando larvas de Pseudaletia unipuncta, Spodoptera frugiperda (Lepidoptera) y Epilachna varivestis (Coleoptera); las tres especies de insectos se rehusaron a comer dietas tratadas con la fracción investigada.

ABSTRACT. Insect antifeeding activity of a fraction obtained from the leaves of Spigelia longiflora (Loganiaceae) was tested on the larvae of Pseudaletia unipuncta, Spodoptera frugiperda (Lepidoptera) and Epilachna varivestis (Coleoptera). All three insect species refused to eat diets treated with the obtained fraction.

Algunas plantas han sido tradicionalmente consideradas como repelentes de insectos. Tal es el caso del pirú, Schinus molle L., cuyas hojas son utilizadas para alejar insectos caseros en algunas regiones del estado de Hidalgo. La búsqueda sistemática de plantas con esas características se ha llevado a cabo desde hace tiempo (Swingle et al., 1941; Sievers et al., 1949). Actualmente en nuestro país algunas instituciones desarrollan proyectos tendentes a encontrar vegetales con propiedades insecticidas y antialimentarias (Arenas, 1984). Para extractos crudos derivados de plantas, las actividades que se reportan son diversas. Así, se encuentran extractos que disuaden la oviposición (Tingle y Mitchell, 1984) o son fotoovicidales (Kagan y Chan, 1983). Algunos son fagoestimulantes (Bristow et al., 1979), otros estimulan la oviposición y la ovogénesis (Monge, 1983) o bien presentan propiedades antialimentarias (Ladd et al., 1978; Chiu, 1983). Esta última propiedad se sospechó en Spigelia longiflora Mart. et Gal. (Loganiaceae), pues en las colectas que de la especie se han hecho no se han observado señales aparentes de daño en el follaje. El objetivo de este estudio es comprobar la actividad antialimentaria de S. longiflora.

${ }^{1}$ División de Investigación Científica y Tecnológica, Universidad Autónoma de Hidalgo, Abasolo 600, c.p. 42000 Pachuca, Hgo.

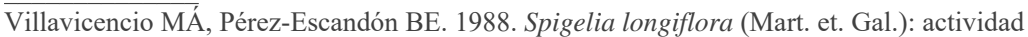
antialimentaria en tres especies de insectos fitófagos. Boletín de la Sociedad Botánica de México 48: $135-138$. 


\section{MATERIAL y MÉTODO}

Se obtuvo un extracto metanólico de hojas frescas de S. longiflora; éste se extrajo varias veces con cloroformo. La capa superior que constituye la Fase Acuosa I (FAI), es la fracción que se utilizó en todas las pruebas.

Se hicieron tres bioensayos diferentes correspondientes a tres especies de insectos que se investigaron.

Experimento 1. Las larvas de Pseudaletia unipuncta (Haworth) (Lepidoptera: Noctuidae) se colectaron en campos de maíz (Zea mays L.) infestados con esta plaga. Para el lote control se usaron tres semillas de maíz, las cuales fueron sumergidas en agua durante cinco segundos, después se colocaron con una larva en un cristalizador pequeño. En el lote experimental las semillas fueron sumergidas en una solución de FAI $1 \%$ y colocadas de igual forma; se hicieron 10 replicados por lote. Después de 16 horas se registraron las semillas y se contó el número de ellas que fueron totalmente consumidas.

Experimento 2. La actividad antialimentaria también se ensayó en Spodoptera frugiperda (J.E. Smith) (Lepidoptera: Noctuidae); en este caso se usaron larvas de $4^{\circ}$ instar. Se procedió de la manera siguiente: en una caja de Petri de $9 \mathrm{~cm}$ de diámetro y $2 \mathrm{~cm}$ de altura se colocó un círculo de papel Whatman 1 el cual fue humedecido con $1 \mathrm{ml}$ de agua destilada. En la caja se colocaron cuatro discos de $0.8 \mathrm{~cm}$ de diámetro preparados con una dieta artificial (Vázquez, 1975) que es usada para alimentar a estos animales. De los dis$\cos$ (control) fueron preparados al mezclar la dieta con agua y los otros dos (experimentales) estuvieron constituidos de la dieta con FAI a tres concentraciones: $0.5,1$ y 2 por ciento. Se colocaron dos larvas por caja. Para cada concentración se hicieron cinco replicados. El experimento se repitió dos veces a la temperatura del laboratorio. Al cabo de 24 hs se contaron las excretas individuales encontradas en las inmediaciones de cada disco. Se considera que éstas provienen del consumo del disco del que se encuentran más cerca. Esto se basa en el hecho de que las larvas de S. frugiperda se desplazan poco al estarse alimentando, de tal forma que sus excreciones se depositan prácticamente en el mismo lugar (M.A. Villavicencio, observación personal). Con este procedimiento se midió el consumo de los discos; a mayor número de excretas, mayor consumo.

Experimento 3. En el caso de Epilachna varivestis (Mulsant) (Coleoptera: Coccinellidae) las larvas que se utilizaron fueron de $4^{\circ}$ instar. Las cajas de Petri que se utilizaron se prepararon igual que para S. frugiperda. Para $E$. varivestis los discos se obtuvieron de hojas de frijol (Phaseolus vulgaris L.) con un horadador de tapones de $2.1 \mathrm{~cm}$ de diámetro. Los discos foliares control se sumergieron por cinco segundos en agua destilada y los experimentales se sumergieron en una solución de FAI a tres concentraciones $(0.5$, 1 y 2 por ciento). Se colocaron dos larvas por caja y se hicieron cinco replicados para cada concentración. El experimento se repitió dos veces. Transcurridas 3 horas se midió el área consumida de cada disco de la siguiente manera: se cortó un disco de papel milimétrico del mismo diámetro, cada disco foliar se colocó sobre el papel y al microscopio estereoscópico se contaron los cuadros completos o incompletos que se observaron. Cada uno se consideró una unidad consumida. 
Los datos obtenidos en cada bioensayo se presentan como Índice de Actividad Antialimentaria (\%) de acuerdo con la siguiente fórmula:

Índice de Actividad Antialimentaria $(\%)=100-\left[\frac{\overline{\mathrm{X}} \text { consumo del control }}{\overline{\mathrm{X}} \text { consumo del experimental }} 100\right]$

El promedio corresponde a los datos de todos los experimentos que se llevaron a cabo con cada especie.

\section{RESULTADOS Y DISCUSIÓN}

Los resultados se resumen en la tabla 1. La actividad antialimentaria de FAI en $P$. unipuncta se ensayó una sola vez a una concentración; sin embargo, la actividad se demuestra claramente, pues ninguna de las semillas tratadas con FAI fue comida y por el contrario, todas las del lote control fueron completamente consumidas. Más aún, las larvas tratadas puparon antes que comer semillas con FAI.

TABLA 1. Actividad antialimentaria de FAI en las tres especies

de insectos investigados. Los valores corresponden al Índice de Actividad Antialimentaria (ver texto)

\begin{tabular}{lrrr}
\hline & \multicolumn{3}{c}{ Concentración de FAI (\%) } \\
\cline { 2 - 4 } Especies & 0.5 & 1.0 & 2.0 \\
\hline P. unipuncta & $*$ & 100.0 & $*$ \\
S. frugiperda & 81.6 & 92.9 & 90.8 \\
E. varivestis & 2.0 & 86.7 & 90.8 \\
\hline
\end{tabular}

* No se ensayó.

La actividad antialimentaria en S. frugiperda, evaluada con base en el número de excretas producidas, también es alta a las tres concentraciones ensayadas; sin embargo los resultados entre las tres no difieren significativamente de acuerdo con la aplicación de la prueba de Kruskal-Wallis (Smith, 1971), ( $\mathrm{P}>0.9)$. A las 24 hs las cajas claramente mostraron que los discos control habían sido más consumidos; los discos tratados habían perdido su forma debido a que habían recibido la visita de las larvas, pero prácticamente no presentaron señales de consumo. Esta situación se mantuvo hasta 60 hs e inclusive, en algunos casos, los discos control fueron totalmente consumidos sin que hubiese consumo aparente de los discos tratados.

En $E$. varivestis la actividad antialimentaria se pierde a la concentración más baja ensayada; sin embargo, a partir de la concentración de $1 \%$ hay actividad (tabla 1) y ésta es considerablemente alta. Por medio de la prueba de "U" de Mann-Whitney (Schwartz, 1980) se comprobó que los resultados obtenidos entre los ensayos efectuados a 1 y 2 por 
ciento no difieren significativamente $(\mathrm{P}>0.05)$. Por otro lado, la protección que FAI confiere a los discos tratados es relativa, pues éstos son consumidos dentro de las 24 hs siguientes cuando ya no tienen otra opción. En este caso FAI se comporta como "antialimentario relativo", siguiendo el término propuesto por Hosozawa et al. (1974) para designar sustancias que retardan el consumo por un período definido de tiempo.

Los resultados que aquí se presentan demuestran claramente la actividad antialimentaria de FAI derivada de S. longiflora en las tres especies de insectos en que se ha probado. Es interesante hacer notar que estas especies aparecen en la lista de plagas del maíz y del frijol en el Reporte Fitosanitario de la SARH (1984) y que FAI, con ensayos previos de invernadero y de campo, puede ser considerada como una alternativa potencial para el control de estas plagas.

AgradeCIMIENTOS. Se agradece al Dr. A. Lagunes del Centro de Entomología y Acarología del CP de Chapingo por las larvas de S. frugiperda donadas y al Sr. Lauro Hernández del Insectario del mismo Centro por su contribución con larvas de $E$. varivestis. Igualmente, nuestro agradecimiento al biólogo Maximino Melgar por el acceso a los Reportes Fitosanitarios de su institución. El presente trabajo fue subsidiado por el Consejo Nacional de Ciencia y Tecnología.

\section{LITERATURA CITADA}

ARENAS, L.C. 1984. Extractos acuosos de polvos vegetales con propiedades insecticidas: una alternativa por explotar. Resúmenes del IX Congreso Mexicano de Botánica, México, pp. 240-241.

Bristow, P.R., R.P. Doss y R.L. CAMPBELL. 1979. A membrane filter bioassay for studing phagostimulatory materials in leaf extracts. Ann. Entomol. Soc. Amer. 72(1):16-18.

CHIU, S.F. 1983. Experiments on the use of seed oils of some meliaceous plants as antifeedants in brown planthopper control. Acta Entomol. Sinica 26(1):1-9.

Hosozawa, S., N. Kato y K. Munakata. 1974. Antifeeding active substances for insects in Caryopteris divaricata. Maxim. Agr. Biol. Chem. 38(4):823-826.

Kagan, S. y G. Chan. 1983. The photoovicidal activity of plant components towards Drosophila melanogaster. Exp. 39:402-405.

LADD, T.L., M. JACOBSON y C.R. BuRIFF. 1978. Japanese beetles: extracts from neem tree seeds as feeding deterrents. J. Econ. Entomol. 71(5):810-813.

Monge, J.P. 1983. Les effets de différents extraits de la plante hôte (Phaseolus vulgaris L.) sur la ponte et l'ovogenèse de la Bruche du haricot (Acanthoscelides obtectus Say). C.R. Acad. Sc. Ser. III Vie 296(12):559-562.

SCHWARTZ, D. 1980. Méthodes statistiques à l'usage des médecins et des biologistes. Flammarion Médecine-Sciences. París.

Secretaría de Agricultura y Recursos Hidráulicos. 1984. Representación General en el Estado de Hidalgo. Subprograma Sanidad Vegetal. Reporte Fitosanitario (marzo-agosto). Pachuca, Hidalgo.

Sievers, W.A. Archer, R.H. Moore y E.R. McGovram. 1949. Insecticidal tests of plants from tropical America. J. Econ. Entomol. 42:549-551.

SMITH, M. 1971. Estadistica simplificada para psicólogos y educadores. Ed. El Manual Moderno, México.

Swingle, M.C., A.M. Phillips y J.B. Gahan. 1941. Laboratory testing of natural and synthetic organic substances as insecticides. J. Econ. Entomol. 34(1):95-99.

Tingle, F.C. y E.R. MitChell. 1984. Aqueous extracts from indigenous plants as oviposition deterrents for Heliothis virescens (F). J. Chem. Ecol. 10(1):101-113.

VÁzQUEZ, G. 1975. Cría masiva del gusano cogollero Spodoptera frugiperda y evaluación de infestación artificial sobre maíz en el campo. Tesis de maestría, Universidad Autónoma de Chapingo, México. 